\title{
High Performance Ion Exchange Membrane for Industrial Use
}

\author{
Kimihiko SATo and Haruhisa MiYAKE* \\ Research Center, Asahi Glass Co., Ltd., \\ 1150 Hazawa-cho, Kanagawa-ku, Yokohama, Japan
}

(Received November 26, 1990)

\begin{abstract}
Salt brine electrolysis using perfluorinated ion exchange membranes is now recognized worldwide as the most energy saving process for the production of chlorine and caustic alkali. Membranes are based on perfluorinated polymers having functional groups.

Although perfluorosulfonated membranes were first made by the du Pont Company, they were not always found to show sufficient performance in chlor alkali process. Asahi Glass succeeded in the development of new membranes through the first successful synthesis of perfluorocarboxylated polymers. These membranes achieved high permselectivity due to less water uptake of the carboxylates as compared with the sulfonates. Currently, many commercial membranes contain layers of both sulfonate and carboxylate perfluoropolymers.

This paper deals with synthetic methods for fluorocarbon acid monomers and polymers, structure and properties of the polymers and membranes made thereof, and industrial applications.

KEY WORDS Salt Brine Electrolysis / Ion Exchange Membrane / Perfluorosulfonated Membrane / Perfluorocarboxylated Membrane /Chlor Alkali Process
\end{abstract}

Polymeric fluorocarbon acids have attracted much attention both industrially and academically in recent years. One main reason is that these substances have come to be known as the only available material which can be used as ion exchange membranes in the chlor-alkali industry-one of the biggest chemical industries.

The membrane chlor-alkali process has made remarkable progress in this decade and is now recognized worldwide as the most energy saving process for the production of chlorine and caustic alkali.

Particularly in Japan, the mercury process withered out by 1986 , having been converted mostly to the membrane process. During the course of the conversion, the membrane process has demonstrated the ability to attain drastic improvements not only in pollution control but also in electrical power consumption. The key of this technology is high performance membranes which are made of perfluorinated polymers having functional groups. 
Polymeric perfluorosulfonic acids were first made by the du Pont Company. These are copolymers of tetrafluoroethylene with perfluorovinyl ethers having sulfonic acid groups. Although the copolymers were originally developed for the use as solid polymer electrolytes for proton transfer in fuel cells, studies on application to a chlor-alkali cell were soon conducted worldwide. As the development proceeded, however, sulfonated perfluoropolymers were not always found to show sufficient performance.

This was considered to be resulted from high water affinity of the polymers due to the presence of strong sulfonic acid group. Thus, perfluorocarboxylic acid polymers were investigated in order to improve the permselectivity. The first successful synthesis of them was made by Asahi Glass Co., Ltd.

This paper deals with preparation of polymers, structure and properties of the polymers and membranes made thereof, and industrial applications.

Preparation of polymers

Perfluorocarboxylated polymers which we newly synthesized have general formulae as shown below. These polymers are copolymers of tetrafluoroethylene and perfluorovinyl ether having carboxylated group instead of sulfonated group. Typical functional monomers were methyl perfluoro-5-oxa-6-heptenoate, methyl perfluoro-6-oxa-7-octenoate and methyl perfluoro-5,8-dioxa-6-methyl-9-decenoate. Herein-after, these monomers are referred to as $M_{1}, M_{2}$ and $M_{3}$, respectively.

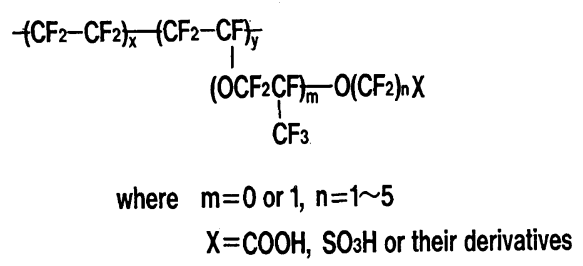

S $\mathrm{CF}_{2}=\underset{\mathrm{CFOCF}_{2} \mathrm{CFO}_{1}\left(\mathrm{CF}_{2}\right)_{2} \mathrm{SO}_{2} \mathrm{~F}}{\mathrm{CF}_{3}}$

$\mathrm{M}_{1} \quad \mathrm{CF}_{2}=\mathrm{CFO}\left(\mathrm{CF}_{2}\right)_{3} \mathrm{COOCH}_{3}$

$\mathrm{M}_{2} \quad \mathrm{CF}_{2}=\mathrm{CFO}\left(\mathrm{CF}_{2}\right)_{4} \mathrm{COOCH}_{3}$

$\mathrm{M}_{3} \quad \mathrm{CF}_{2}=\mathrm{CFOCF}_{2} \mathrm{CFO}_{1}\left(\mathrm{CF}_{2}\right)_{3} \mathrm{COOCH}_{3}$

A typical preparative route to carboxylated perfluorovinyl ethers is shown here.

$$
\begin{aligned}
& \mathrm{CF}_{2}=\mathrm{CF}_{2}+\left.\mathrm{I}_{2} \longrightarrow \mathrm{I}\left(\mathrm{CF}_{2} \mathrm{CF}_{2}\right)_{2} \mathrm{I} \stackrel{\text { oleum }}{\longrightarrow} \underset{\mathrm{O}}{\mathrm{CF}_{2}}\right|_{\mathrm{C}=0} ^{\mathrm{CF}} \\
& \stackrel{\mathrm{CH}_{3} \mathrm{OH}}{\longrightarrow} \mathrm{FOCCF}_{2} \mathrm{CF}_{2} \mathrm{CO}_{2} \mathrm{CH}_{3} \stackrel{(x+1) \mathrm{HFPO}}{\longrightarrow}
\end{aligned}
$$

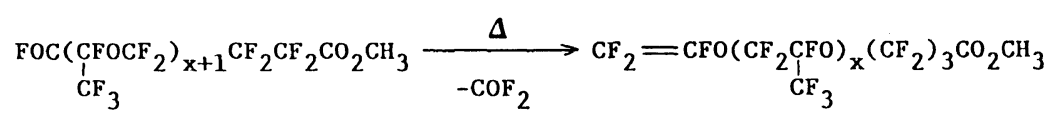

$$
\begin{aligned}
& (x=0,1)
\end{aligned}
$$


A cyclic lactone is formed in considerable yield (70-80\%) by the reaction of 1,4-diiodo-perfluorobutane with oleum. The controlled addition of methanol to the lactone gives selectively 3-methoxycarbonyl perfluoropropionyl fluoride, to which hexafluoropropylene oxide is added.

Finally, the acid fluoride group is converted to a perfluorovinyl group by pyrolysis. An alternative pathway from the lactone to the vinyl ether has been developed to improve the yield.

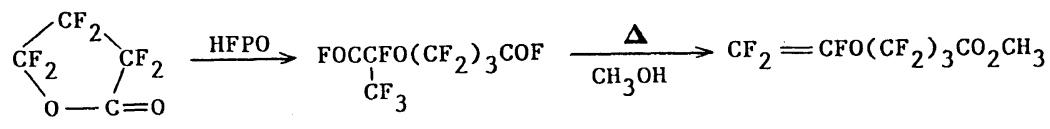

The molar ratio of functional perfluorovinyl ether to tetrafluoroethylene in copolymer is directly related to ion exchange capacity of the resulting polymeric acid. This ratio can be controlled by monomer feed ratio in the copolymerization procedure. Since perfluorovinyl ethers have been known to be radically active, copolymerization of tetrafluoroethylene and functional perfluorovinyl ether was carried out by using a radical initiator.

Fig.1 shows the copolymer composition curve obtained for the copolymerization of tetrafluoroethylene and functional monomers $\left(\mathrm{M}_{1}\right.$ and $\mathrm{M}_{3}$ ). The curve in this figure clearly indicates that both monomers have almost the same reactivity toward tetrafluoroethylene irrespective of the difference of side chain length. The monomer reactivity ratios of tetrafluoroethylene and functional vinyl ethers were calculated as 7.0 and 0.14 , respectively, by using Fineman-Ross Method. From these reactivity ratios, $Q$ and $e$ values of each monomer were derived by the Alfrey-Price equation. Since $Q$ and $e$ values of tetrafluoroethylene are known to be 0.049 and $1.22, Q$ and $e$ values of functional vinyl ethers were obtained as 0.0057 and 1.08 , respectively. The much smaller $Q$ value of these vinyl ethers as compared with that of tetrafluoroethylene explains their lower reactivity than that of tetrafluoroethylene.

\section{Characterization}

Characterization of polymers were carried out for copolymers of tetrafluoroethylene and $M_{1}$. Fig.2 shows a typical IR spectrum.

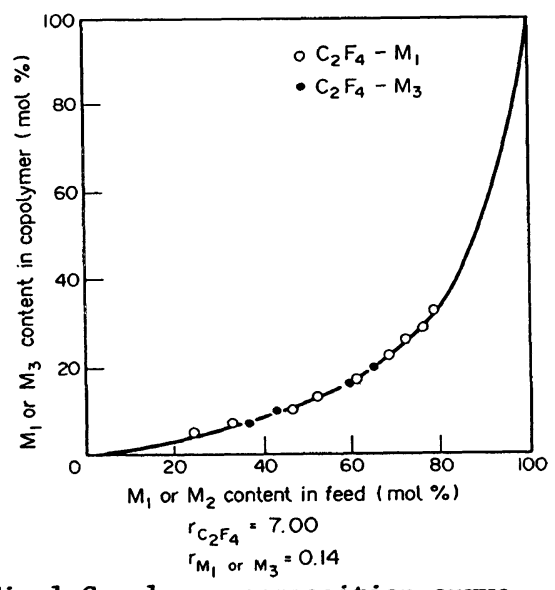

Fig.1 Copolymer composition curve

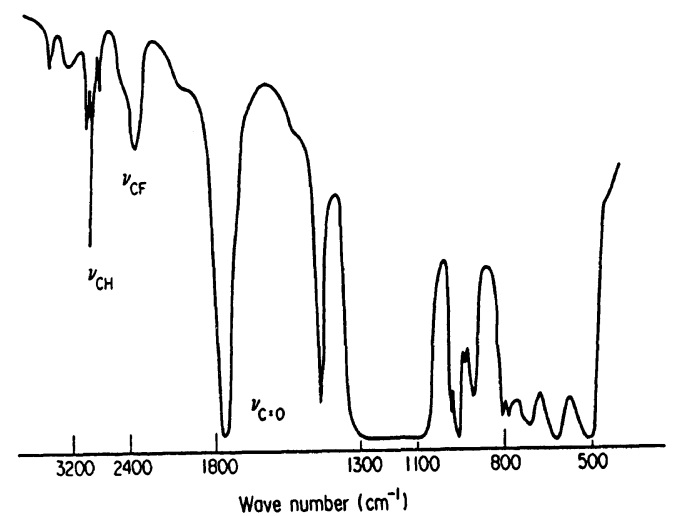

Fig.2 IR spectrum of copolymer 
The absorption at 2960 and $1780 \mathrm{~cm}^{-1}$ are due to carbon-hydrogen bond and carbonyl bond in ester group, respectively. The absorption in the range of $1100 \sim 1300 \mathrm{~cm}^{-1}$ are assigned to carbon-fluorine bonds, which appear commonly in fluorocarbon polymers. The formation of copolymers was also confirmed by fluorine NMR spectrum giving their characteristic peaks as shown in Fig. 3.

Whereas methyl ester type copolymers were insoluble in common solvents, decyl ester type copolymers were soluble in fluorocarbon solvents such as chlorbenzotrifluoride. Thus, molecular weight of copolymers were measured by using an osmotic pressure method and found to be in the range of $105 \sim 106$. In Fig.4, molecular weight is plotted against $T_{Q}$ value which is defined as the temperature at which the volumetric melt flow rate of copolymer is $100 \mathrm{~mm}^{3} / \mathrm{sec}$ through the orifice of $1 \mathrm{~mm}$ diameter and $1 \mathrm{~mm}$ length under the load of $30 \mathrm{~kg} / \mathrm{cm}^{2}$. As shown in this figure, logarithmic molecular weight has good linear relationship with $T_{Q}$ value. Crystallinity of copolymers was measured by the wide angle $\mathrm{X}$-ray diffraction method as shown in Fig.5. It decreased with increasing functional vinyl ether content and the copolymer became almost amorphous above the vinyl ether content of 20 mole\%.

Furthermore, copolymers were melt processable as shown in Fig.6.

\section{Properties}

Copolymers are used in the field of ion permselective membranes by taking advantage of ion exchange functions. Accordingly, properties as ion exchange membranes are important from the practical point of view.

Most important and fundamental properties of membranes are mechanical strength, ion permselectivity and conductivity. These properties strongly depended upon the degree of dissociation of functional group and cohesive forces of fluorinated polymer chains, which are related to the type of functional groups and molecular structure of polymers, respectively.

Figure 7 shows a stress-strain curves of the perfluorocarboxylated membrane in the state of ester and sodium salt. The sodium type membrane exhibited significant increase of tensile modulus and decrease of elongation as compared with ester type one. This indicates the formation of some kind of crosslinking structure by incorporation of ionic moieties. Fig. 8 shows the small angle X-ray scattering pattern. The appearance of a new peak in the sodium salt type membrane strongly suggests the ionic cluster formation.

The shift of peaks to lower angle was observed by diluting the concentration of the soaked solution. The formation of cluster in perfluorinated ion exchange membranes were confirmed by several researchers.

Table 1 shows the temperature dependence of tensile strength. The sodium type membrane retains its high strength even at a elevated temperature of $90^{\circ} \mathrm{C}$, although the ester type membrane loses its strength with the rise of temperature. These behavior are also explained by ionic clustering.

Table 1 Tensile Strength of the Membranes.

In the membrane, there exists fixed ion concentration which is defined as miliequivalent weight of functional group per unit weight of 
High Performance Ion Exchange Membrane for Industrial Use

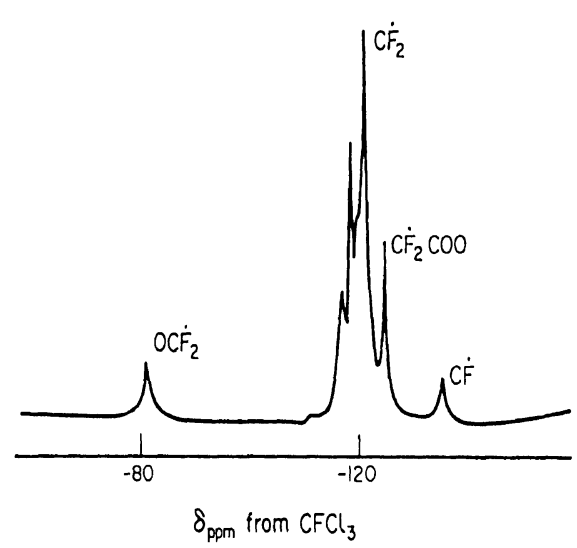

Fig. ${ }^{19}$ F-MMR spectrum of copolymer

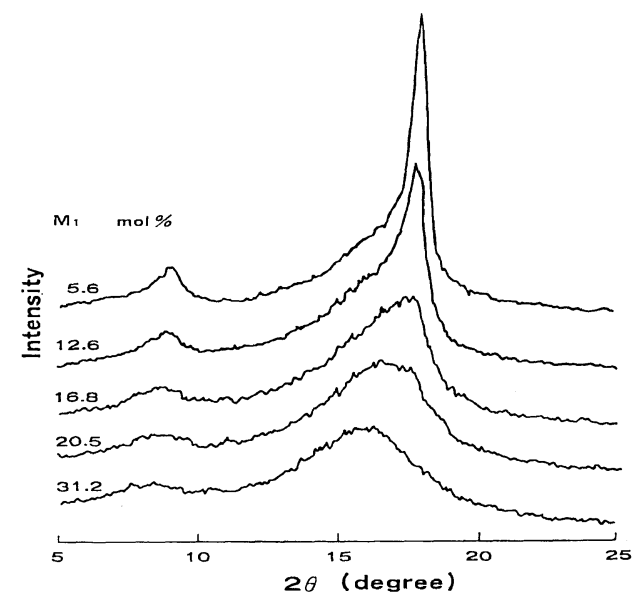

Fig.5 X-ray spectra of copolymers

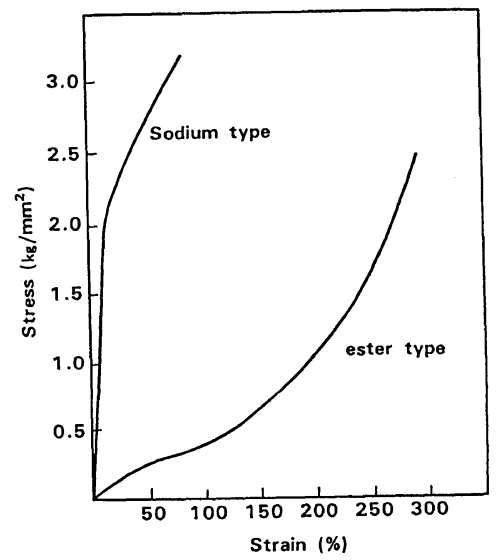

Fig.7 Stress-strain curves of the membranes

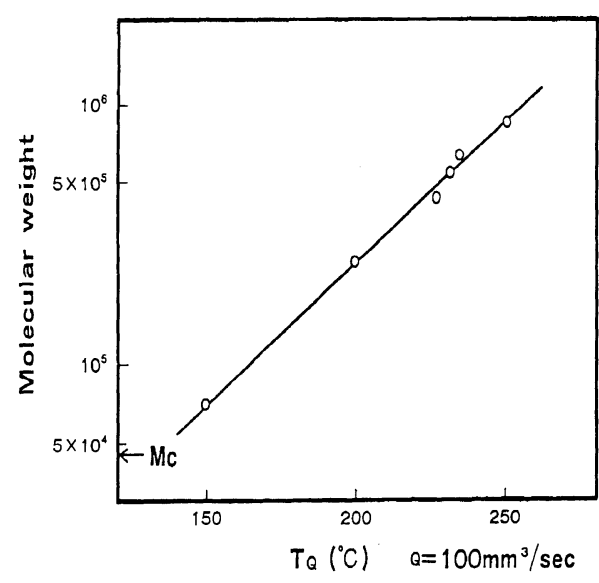

Fig.4 Molecular weight and $\mathrm{T}_{Q}$

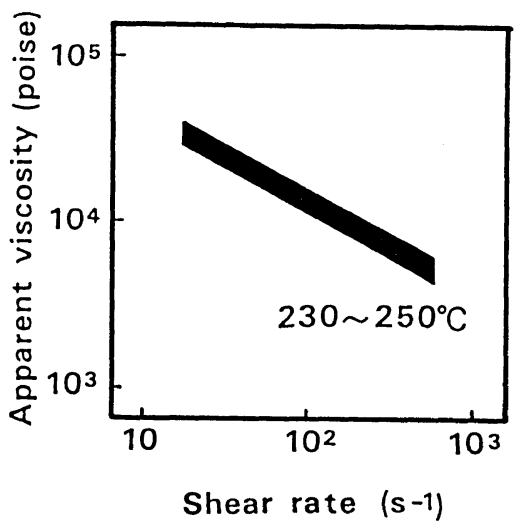

Fig.6 Melt flow behavior of polymer

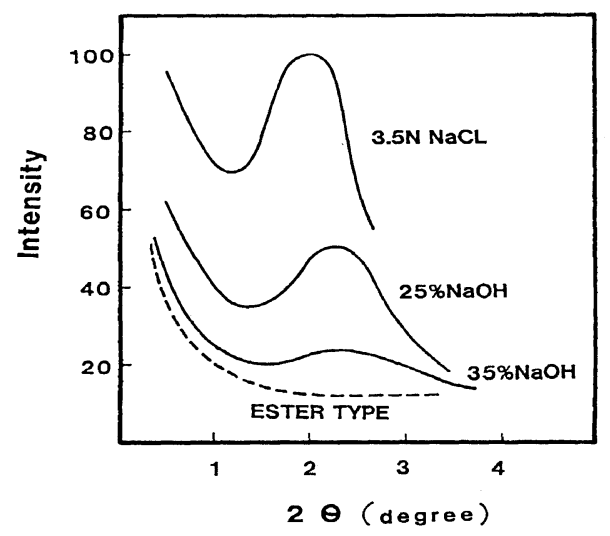

Fig.8 Small angle X-ray scatterings of Na salt type membrane 
water. Figure 9 shows the relationship between ion exchange capacity and water content of different molecular type of polymers including both perfluorocarboxylic and perfluorosulfonic acid ones. It is shown that water content increases with the increase of ion exchange capacity for each membrane. However, the slopes are much smaller with carboxylic type membranes than that with sulfonic acid type membrane and the former show lower water content than the latter. Among carboxylated membranes, the one having shorter side chain length exhibited much lower water content.

Since carboxylated membranes have low water content even with high ion exchange capacity, they can attain considerably high fixed ion concentration expressed as Aw.

Figure 10 shows fixed ion concentration of various membranes which are soaked in caustic soda. As expected from the above facts, perfluorocarboxylate membranes have higher fixed ion concentration than perfluorosulfonate membranes, and the difference is remarkable particularly in high caustic concentration rage.

Figure 11 shows hydroxyl ion concentration in Donnan equilibrium in membrane with that of outer caustic solution. As is clear in this figure, perfluorocarboxylate membranes have lower value of Donnan hydroxyl ion concentration than perfluorosulfonate membranes, because of the higher fixed ion concentration of the former. These features of perfluorocarboxylated membranes directly result in higher ion permselectivity leading to the higher current efficiency than perfluorosulfonated membranes when they are used in chlor-alkali electrolytic process.

Fig.12 shows current efficiency obta-ined with perfluorocarboxylated memb-rane in a chlor-alkali cell.

Current efficiency as high as $95 \sim 97 \%$ is attained in high caustic solution, whilst such a high current efficiency is never obtained with perfluorosulfonated membrane.

Figure 13 shows the electric conductivity for both carboxylic and sulfonic acid membranes. Conductivity increases with the increase of ion exchange capacity. At the same level of ion exchange capacity carboxylic acid membranes have lower conductivity than sulfonic acid ones, because dissociation constant of carboxylic acid is lower than that of sulfonic acid. However, carboxylic acid membranes can attain high conductivity by increasing ion exchange capacity. In the meanwhile, conductivity was affected not only by molecular structure but also by environmental conditions in which membranes are soaked. Dependence of the conductivity upon concentration of caustic soda at several temperatures is shown in Fig.14 in case of perfluorocarboxylate membranes.

The conductivity is seen to be strongly affected by the caustic concentration and the temperature. From the figure, the apparent activation energy for the ionic conductance was calculated to be $3.9,9.1$ and $17.1 \mathrm{kcal} / \mathrm{mol}$ in 14,25 and $40 \mathrm{wt} \%$ caustic solution, respectively. The decrease of conductivity with the increase of caustic concentration is ascribed to the decrease in ionic mobility, which is caused by the loss of water in the membrane. The low value of the conductivity and the high value of the activation energy in high caustic solutions implies that there exists a strong interaction between counter ion and fixed ion in the membrane.

On the otherhand, high ion exchange capacity membranes can serve as high conductive membranes even in high caustic concentrations. 
High Performance Ion Exchange Membrane for Industrial Use

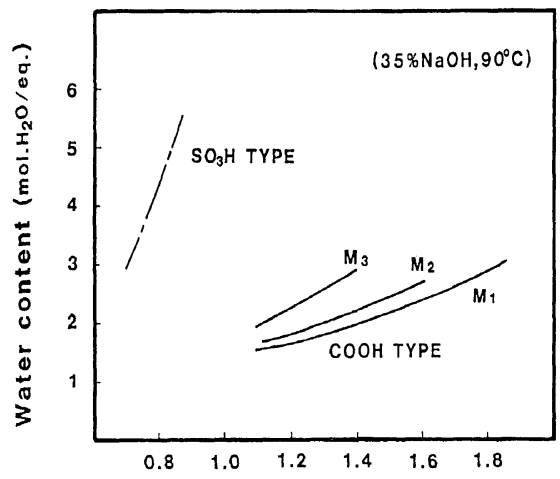

Ion exchange capacity (meq/g-dry polymer)

Fig.9 Dependence of water content upon ion exchange capacity of membranes

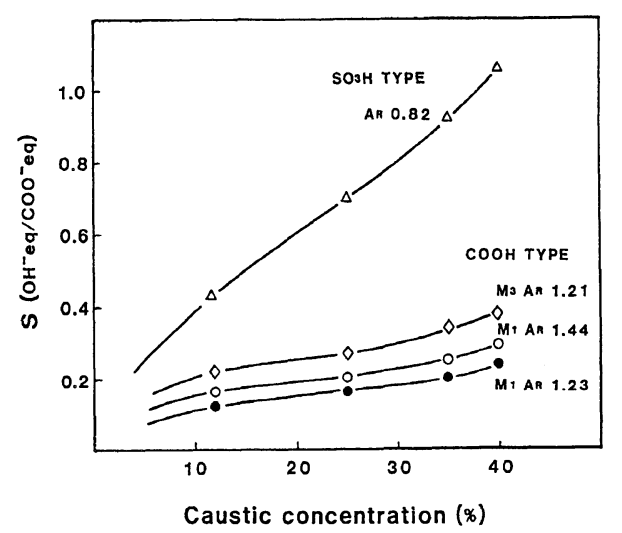

Fig.11 S value and caustic concentration

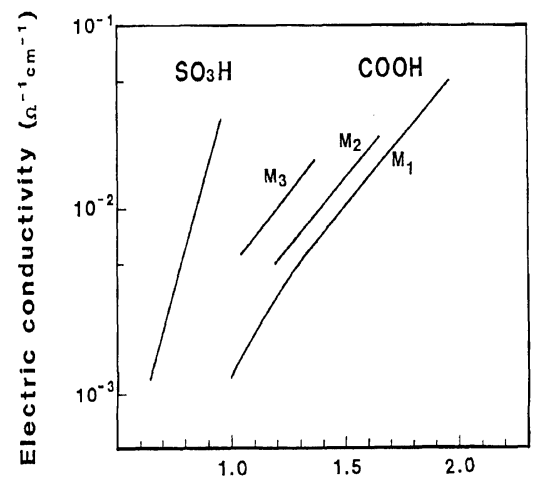

Ion exchange capacity (meq/g-dry polymer)

Fig.13 Conductivity of membranes against ion exchange capacity

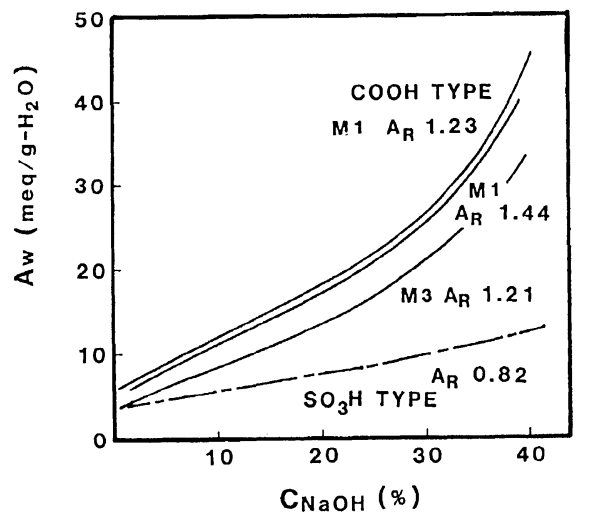

Fig.10 Influence of caustic concentration upon fixed ion concentration

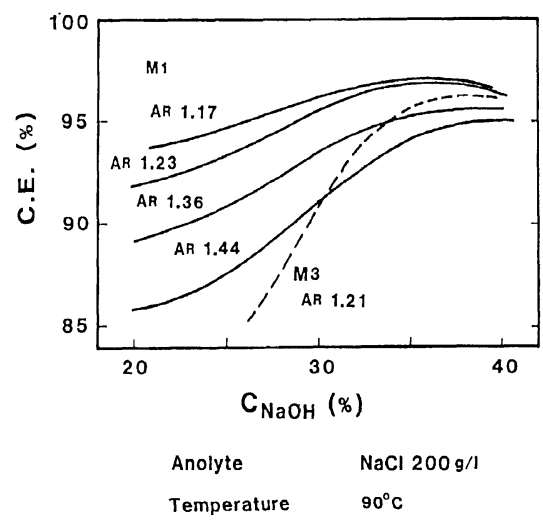

Fig.12 Influence of caustic concentration upon current efficiency

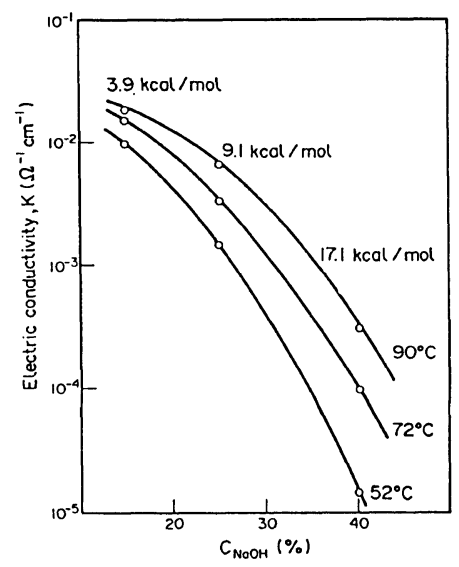

Fig. 14 Dependence of membrane conductivity upon caustic concentration 
Table 2 shows the activation energy and conductivity of membranes in $35 \%$ caustic solution against ion exchange capacity.

As is clear in this table, high ion exchange capacity can weaken the ionic interaction effectively.

Diffusion of ions through perfluorocaboxylic acid membranes has been studied. A measurement cell is divided by a membrane into two compartments which are filled with caustic and sodium chloride solution respectively. As shown in Fig.15 and Fig.16, apparent diffusion coefficients of $\mathrm{NaCl}$ and $\mathrm{NaOH}$ depend strongly upon caustic concentration, while their dependence upon $\mathrm{NaCl}$ concentration is smaller. When the concentration of both solutions is equal, for example $3.5 \mathrm{~N}$, the apparent diffusion coefficient of sodium hydroxide in the membrane is almost 5 times as large as that of sodium chloride. In the meanwhile, diffusion coeffifients of $\mathrm{NaOH}$ and $\mathrm{NaCl}$ in aqueous solutions of the same concentration are almost the same and are in the range of $10-5 \mathrm{~cm}^{2} / \mathrm{sec}$. This means that their diffusion coefficients in membrane lower remarkably but that the coefficient of $\mathrm{NaOH}$ is not affected so much as that of $\mathrm{NaCl}$.

Meanwhile, there should be a correlation between conductivity and diffusion coefficinent, because both conductivity and diffusibility are based on ionic mobility in a membrane. Figure 17 presents a relationship

Table 2 Activation energy and ionic conductance

of membranes

$35 \% \mathrm{NaOH}$

\begin{tabular}{|rr|c|c|c|c|}
\hline Ion Exchange capacity & 1.2 & 1.4 & 1.7 & 1.9 \\
$(\mathrm{meq} / \mathrm{g})$ & & 14.4 & 12.0 & 10.4 \\
\hline $\mathrm{Ka} \quad(\mathrm{kcal} / \mathrm{mol})$ & 15.3 & 14.3 & 8.5 & 87 & 300 \\
\hline
\end{tabular}

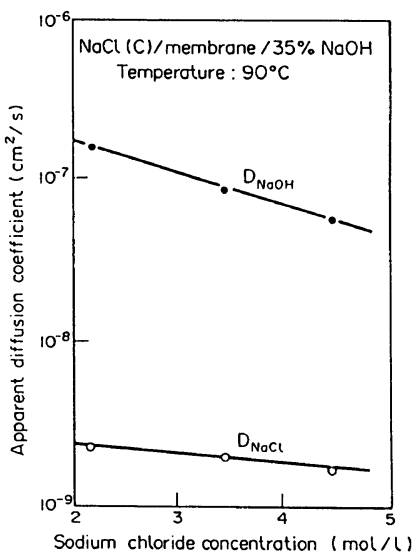

Fig.16 Dependence of diffusion coefficient upon $\mathrm{NaCl}$ concentration

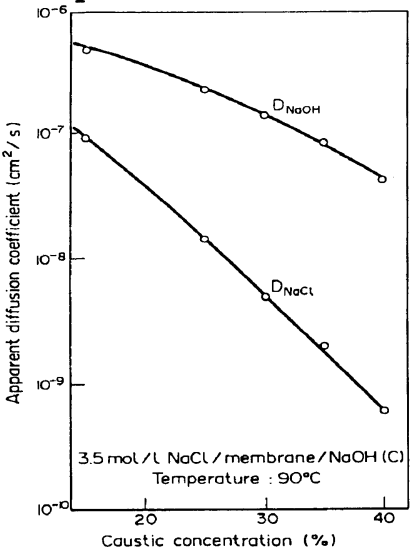

Fig.15 Dependence of apparent diffusion coefficient upon $\mathrm{NaOH}$ concentration

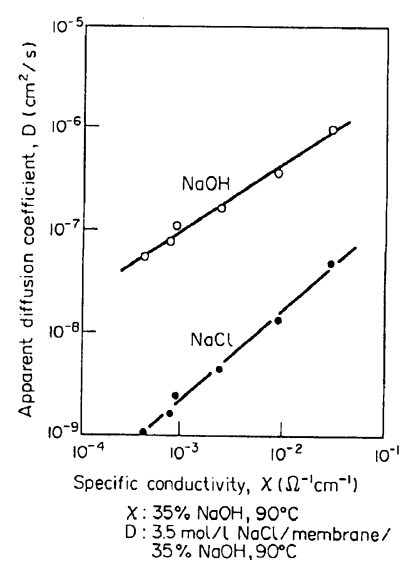

Fig.17 Correlation between diffusion coefficient and membrane conductivity 
between conductivity and diffusion coefficients of electrolytes. Transfer of ions under current passage is also important. Figure 18 shows flux of sodium chloride with and without current passage. The influence of current passage upon sodium chloride flux was noteworthy. The decrease in the flux is caused by the draw-back of chloride anion to the anode side.

In this figure, the broken line shows the value measured without current passage in which the flux of sodium chloride is 3-8 times as large as that with current passage. The fact that the flux of sodium chloride with current passage decreases with increasing

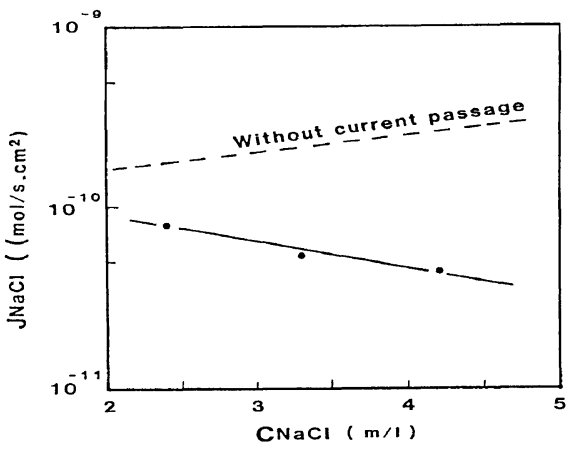

$\oplus \mathrm{NaCl}$ (c)/Membrane/NaOH $35 \% \ominus$ Current Temperature : $90^{\circ} \mathrm{C}$

sodium chloride concentration indicates complexity of material transfer.

Application to the chlor alkali process

The most important and large-scale application of polymeric perfluorinated acids is for the membrane in the chlor alkali process.

Fig.19 is a basic illustration of membrane cell technology. The membrane divides the cell into anode and cathode chambers.

Saturated brine is fed to the anode chamber and chlorine is generated at the anode. Water is fed to the cathode chamber and produces hydrogen and hydroxide ions, the latter of which combine with the sodium ions to form sodium hydroxide. The electrochemical reactions for the membrane all are given as;

$$
\begin{array}{ll}
\text { anode } & 2 \mathrm{NaCl} \rightarrow \mathrm{Cl}_{2}+2 \mathrm{Na}^{+}+2 \mathrm{e} \\
\text { cathode } & 2 \mathrm{H}_{2} \mathrm{O}+2 \mathrm{e} \rightarrow \mathrm{H}_{2}+2 \mathrm{OH}^{-} \\
\text {overall } & 2 \mathrm{NaCl}+2 \mathrm{H}_{2} \mathrm{O} \rightarrow \mathrm{Cl}_{2}+\mathrm{H}_{2}+2 \mathrm{NaOH} .
\end{array}
$$

The membrane permits only the passage of sodium ions from the anode chamber to the cathode chamber and prevents the migration of hydroxyl ions to the anode chamber, which would reduce the current efficiency if it occurred.

The essential requirements for the membrane used in chlor alkali cells are high conductivity, selectivity and mechanical strength.

To achieve both high current efficiency and high conductivity, the membranes are composed of at least two layers.

Perfluorosulfonated polymers or perfluorocarboxylated polymers having high ion exchange capacity are used for the anode side layer, in order to attain high conductivity. Perfluorocarboxylated polymers with low ion exchange capacity are used for the

cathode side layer in order to achieve Fig.19 Principle of a membrane chlor-alkali cell

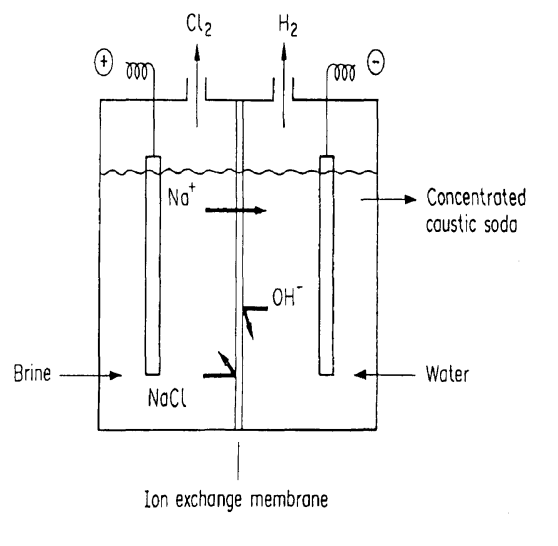


high current efficiency.

To endow the membranes with necessary strength, the membrane are reinforced with polytetrafluoroethylene woven cloth. Furthermore, surfaces of the membranes are coated with hydrophilic inorganic porous layer so as to release gas bubbles being generated in electrolytic cells.

The maximum caustic soda concentration that can be obtained lies in the range of $30 \sim 40 \%$ caustic. The current efficiency is $95 \sim 96 \%$ and an interelectrode voltage of about $2.90 \mathrm{volt}$ is obtained at $35 \%$ caustic concentration under a current density of $30 \mathrm{~A} / \mathrm{dm}^{2}$.

Energy consumption by the membrane process is now approximately $1000 \mathrm{kwt} / \mathrm{t}$ lower than that of the mercury or the diaphragm process ( $30 \%$ energy saving ).

A commercial membrane process came into operation in 1975 and since then, has been increasingly adopted in many countries from the viewpoint of energy saving and pollution control.

World caustic production via the membrane process now amounts to 7.4 million tons per yer and the membrane process accounts for $16 \%$ of installed worldwide caustic capacity. In the year 2000, total production capacity is estimated to reach 55 million tons and at that time, the membrane process is expected to occupy $50 \%$.

The perfluorinated membranes have up to now been used primarily in the chlor alkali industry and membrane technology has advanced to meet the severe requirements in this field. Now that perfluorinated membranes with varied performance have become commercially available, versatile applications in other fields are expected to flourish in the near future. 